\title{
GROUND-STATE STRUCTURE OF A TWO-ELECTRON QUANTUM DOT
}

\author{
M. EL-SAID \\ Department of Physics, Eastern Mediterranean University \\ Gazi Magusa, Mersin 10, Turkey
}

(Received July 20, 1999; revised version February 8, 2000)

\begin{abstract}
The energy spectrum of two interacting electrons, confined in a parabolic quantum dot, presented in a magnetic field is obtained. We have analyzed the energy level crossings between spin-singlet and spin-triplet states. The influence of the quantum dot size on these crossings is also shown. The results of our calculations seem to be in good agreement with analytical and exact numerical data.
\end{abstract}

PACS numbers: 73.20.Dx

\section{Introduction}

Quasi-zero-dimensional systems, such as quantum dots (QDs), have been the subject of intense research in recent years, due to the nanofabrication techniques that make possible the realization of systems of very small dimensions comparable to the de Broglie wavelength of carriers. In such small structures the dynamics of electrons is fully quantized into a discrete spectrum of energy levels. The confinement in $z$-direction, which is the growth direction, is assumed to be stronger than in the $x y$-plane, so that the dot can be viewed as a two-dimensional disk. Different experimental [1-5] and theoretical [8-20] methods have been used to investigate the energy spectrum and the correlation effects of the interacting electrons confined in quantum dots under the effect of an applied magnetic field. One of the most interesting features of the electron correlation is the change of the spin and angular momenta structures in the ground state of those systems in the presence of the magnetic field. The singlet-triplet electron transition that occurs in the two-electron parabolic quantum dot is a simple case but not a trivial one and has recently received much attention [14-17].

In this work we shall study the spectroscopic properties of the two-electron quantum dot using a different approach, namely the shifted $1 / D$ expansion method, where $D$ is the number of spatial dimensions. We progress in two steps: firstly, we used the shifted $1 / D$ expansion to produce an analytical energy expression for 
two interacting electrons confined in a quantum dot in the presence of a magnetic field of arbitrary strength. Secondly, we focus on the spin and angular momenta transitions and give explanation to these phenomena, which occur in the spectra by making use of the energy expression we have obtained. The results of this work are outlined in the following sections. In Sec. 2 we present the Hamiltonian theory for two interacting electrons parabolically confined in quantum dot and obtain the eigenenergy expression using the $1 / D$ expansion method. In Sec. 3 we present our computed results and explanation to the singlet-triplet transition. The conclusion is given in the final section.

\section{Model}

The effective-mass Hamiltonian, $H$, for two interacting electrons, parabolically confined in a quantum dot, in the presence of a perpendicular magnetic field applied parallel to the $z$-axis is given as

$$
\begin{aligned}
H= & \sum_{i=1}^{2}\left[-\frac{\hbar^{2}}{2 m^{*}} \nabla_{i}^{2}+\frac{1}{2} m^{*} \omega^{2} r_{i}^{2}+\frac{\hbar \omega_{c}}{2} L_{i}^{z}\right] \\
& +\frac{e^{2}}{\varepsilon\left|r_{2}-r_{1}\right|}+g^{*} \mu_{\mathrm{B}} B \sum_{i} S_{i, z},
\end{aligned}
$$

$L_{i}^{z}$ and $S_{i, z}$ stand for the $z$-components of the orbital angular momentum and spin of each electron, $\mu_{\mathrm{B}}=e \hbar / 2 m^{*}, g^{*}, \omega_{\mathrm{c}}=e B / m^{*} c$, and $\varepsilon$ are the Bohr magneton, Lande factor, the cyclotron frequency, and the dielectric constant of the medium, respectively. The frequency $\omega$ depends on the magnetic field $B$ and the confinement frequency $\omega_{0}$ and is given as

$$
\omega=\left(\omega_{0}^{2}+\frac{\omega_{c}^{2}}{4}\right)^{1 / 2}
$$

Upon introducing the center of mass $(\mathrm{cm}) \boldsymbol{R}=\left(\boldsymbol{r}_{1}+\boldsymbol{r}_{2}\right) / \sqrt{2}$ and the relative coordinates $r=\left(r_{1}-r_{2}\right) / \sqrt{2}$, the Hamiltonian in Eq. (1) decoupled to the $\mathrm{cm}$ motion Hamiltonian

$$
H_{R}=-\frac{\hbar^{2}}{2 m^{*}} \nabla_{R}^{2}+\frac{1}{2} m^{*} \omega^{2} R^{2}+\frac{\hbar \omega_{\mathrm{c}}}{2} L_{z}^{R}
$$

and the relative motion Hamiltonian

$$
H_{r}=-\frac{\hbar^{2}}{2 m^{*}} \nabla_{r}^{2}+\frac{1}{2} m^{*} \omega^{2} r^{2}+\frac{\hbar \omega_{\mathrm{c}}}{2} L_{z}^{r}+\frac{e^{2}}{\sqrt{2} r} .
$$

Equation (3) describes the Hamiltonian of the harmonic oscillator with well known eigenenergies

$$
E_{n_{\mathrm{cm}}, m_{\mathrm{cm}}}=\left(2 n_{\mathrm{cm}}+\left|m_{\mathrm{cm}}\right|+1\right) \hbar \omega+\frac{\hbar \omega_{\mathrm{c}}}{2} m_{\mathrm{cm}},
$$

labelled by the radial $\left(n_{\mathrm{cm}}=0,1,2, \ldots\right)$ and the azimuthal $\left(m_{\mathrm{cm}}=0, \pm 1, \pm 2, \ldots\right)$ quantum numbers. Antisymmetrization of the two-electron wave function requires that even $m$ states are singlets and odd $m$ states are triplets with the Zeeman energy term $E_{\text {spin }}=g^{*} \mu_{\mathrm{B}} B S_{z}$ and the total spin $S_{z}=\left[1-(-1)^{m}\right] / 2$ each representing a good quantum number for the system. The Zeeman energy is very 
small for QD made from GaAs $\left(g^{*}=-0.44, m^{*}=0.067 m_{\mathrm{e}}\right)$ in an applied magnetic field of practical strength $\sim 10 \mathrm{~T}$. However, the oscillations of the spin make the spectra of the quantum dot more rich. The total energy states of the Hamiltonian $E=E_{R}\left(n_{\mathrm{cm}}, m_{\mathrm{cm}}\right)+E_{r}\left(n_{r}, m\right)+E_{\text {spin }}\left(S_{z}\right)$ are labelled by the $\mathrm{cm}$ and relative quantum numbers $\left|n_{\mathrm{cm}}, m_{\mathrm{cm}} ; n_{r}, m\right\rangle$. The problem is reduced to obtaining the eigenenergies $E_{n_{r}, m}$ of the relative motion Hamiltonian. The eigenenergies are obtained by the help of the $1 / D$ expansion method. In $D$ spatial dimensions the radial Schrödinger equation for the effective potential $V(r)=\frac{\sqrt{2}}{r}+\frac{1}{4} \omega^{2} r^{2}+m \frac{\omega_{c}}{2}$ becomes

$$
\left[-\frac{\mathrm{d}^{2}}{\mathrm{~d} r^{2}}+\frac{(\bar{k}+a-1)(\bar{k}+a-3)}{4 r^{2}}+V(r)\right] \psi(r)=E_{n_{r}, m} \psi(r)
$$

where $\bar{k}=D+2 m-a$ and $a$ is the shift parameter. Following the previous work of the shifted 1/ $D$ expansion method [21-24], we give here only the energy expression which is needed to calculate the spectra of $H_{r}$. The energy expression then reads as

$$
\begin{aligned}
& E_{n_{r}, m}=E_{n_{r}, m}^{(0)}+\frac{\bar{k}^{2}}{4 r_{0}}+\frac{1}{r_{0}^{2}}\left[\frac{(1-a)(3-a)}{4}+\alpha_{1}\right]+\frac{\alpha_{2}}{\bar{k}_{0}^{2}}, \\
& E_{n_{r}, m}^{(0)}=\frac{\sqrt{2}}{r_{0}}+\frac{1}{4} \omega^{2} r_{0}^{2}+m \frac{\omega_{\mathrm{c}}}{2} \\
& a=2-2\left(2 n_{r}+1\right) \omega, \\
& \bar{\omega}=\left[3+r_{0} \frac{V^{\prime \prime}\left(r_{0}\right)}{V^{\prime}\left(r_{0}\right)}\right]^{1 / 2}
\end{aligned}
$$

and the roots $r_{0}$ are determined through the relation

$$
\left[2 e_{0}^{3} V^{\prime}\left(r_{0}\right)\right]^{1 / 2}=2+2|m|-a \text {. }
$$

The explicit forms of the parameters $\alpha_{1}$ and $\alpha_{2}$ are given in the Appendix in terms of $n_{r}, \bar{\omega}, r_{0}$, and $a$. Once the roots $r_{0}$ for a particular quantum state $\left|n_{r}, m\right\rangle$ and the confining frequency $\omega$ are calculated, the task of computing the energy using Eq. (7) is relatively simple.

\section{Results}

Our numerical results are computed for QD made of GaAs|AlGaAs and presented in Figs. 1-3 and Tables I-V. In Fig. 1 we have shown the energies of the states $|0,0 ; 0, m\rangle, m=0,-1,-2, \ldots$ for two interacting electrons parabolically confined in the quantum dot of size $\ell=3 a^{*}$ as a function of the ratio $\omega_{\mathrm{c}} / \omega_{0}$. The numerical results for the states $m=0,-1,-5,-7$ are also given in Table I for the sake of comparison.

As the ratio $\omega_{c} / \omega_{0}$ increases, the energy of the state $m=0$ increases while the energies of the states with non-vanishing azimuthal quantum number $m$ decreases, thus leading to a sequence of different ground states. The transition in the $m$-quantum number is accompanied by a flip in the spin of the state to keep the wave function of the electron state totally antisymmetric in accordance with Pauli 


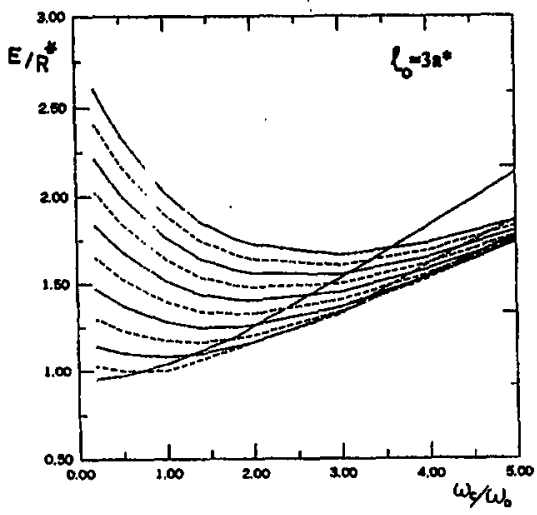

Fig. 1. The total energies of the states $|0,0 ; 0, m\rangle, m=0,-1,-2, \ldots,-10$ for two interacting electrons, parabolically confined in the $\mathrm{QD}$ of size $\ell=3 a^{*}$ and $g^{*}=-0.44$. For GaAs $a^{*}=98.7 \AA$ and $R^{*}=5.83 \mathrm{meV}$ (-

\section{TABLE I}

The energies of the relative states $|0, m\rangle, m=$ $0,-1,-5,-7$ for the quantum dot size $\ell=3 a^{*}$ calculated at different values of $\omega_{\mathrm{c}} / \omega_{0}$ by the $1 / D$ method. Energies are in $R^{*}$.

\begin{tabular}{c|c|c|c|c}
\hline \hline$\omega_{\mathrm{c}} / \omega_{0}$ & $m=0$ & $m=-1$ & $m=-5$ & $m=-7$ \\
\hline 0.2 & 0.953 & 1.013 & 1.655 & 2.02 \\
0.5 & 0.973 & 1.002 & 1.528 & 1.845 \\
1.0 & 1.040 & 1.002 & 1.397 & 1.641 \\
1.4 & 1.115 & 1.065 & 1.341 & 1.540 \\
1.8 & 1.195 & 1.126 & 1.327 & 1.491 \\
2 & 1.256 & 1.162 & 1.330 & 1.481 \\
3 & 1.531 & 1.324 & 1.405 & 1.499 \\
4 & 1.827 & 1.613 & 1.557 & 1.617 \\
5 & 2.130 & 1.865 & 1.741 & 1.777 \\
6 & 2.524 & 2.441 & 3.578 & 4.264 \\
7 & 2.834 & 2.762 & 4.075 & 4.857 \\
8 & 3.141 & 3.083 & 4.578 & 5.561 \\
9 & 3.444 & 3.403 & 5.084 & 6.068 \\
10 & 3.745 & 3.722 & 5.592 & 6.680
\end{tabular}

exclusion principle. We observed from Fig. 1 that the first transition $(m: 0 \rightarrow-1$, $s: 1 \rightarrow 0)$ occurs at $\omega_{c} / \omega_{0} \approx 0.6$ and the second one $(m:-1 \rightarrow-2, s: 0 \rightarrow 1)$ at $\omega_{\mathrm{c}} / \omega_{0} \approx 1.8$. For the fixed value of $\ell_{0}=\left(\hbar / m^{*} \omega_{0}\right)^{1 / 2}$, equivalently $\omega_{0}$, the second transition occurs at a higher $\omega_{c} / \omega_{0}$ ratio. These results are in agreement with those reported in Ref. [15]. The ground state of the interacting electrons confined in the 
quantum dot due to this transition, changes its angular momentum $(m)$ and its spin $(s)$ quantum numbers as shown in the figures.

The transition phenomena can be understood from the competition between Coulomb and magneto-confining energies which appears in Eq. (8). The major contribution $(\sim 50 \%)$ to the relative energy $E_{n_{r}, m}$ of the system comes from the first term, namely,

$$
E_{n_{r}, m}^{(0)}=\frac{\sqrt{2}}{r_{0}}+\frac{1}{4} \omega^{2} r_{0}^{2}+m \frac{\omega_{c}}{2} .
$$

The roots of $r_{0}$ are calculated for all the quantum states $|0,0 ; 0, m\rangle$, $m=0,-1,-2,-3, \ldots$ at different values of the ratio $\omega_{\mathrm{c}} / \omega_{0}$. Various values for the roots are given for $\omega_{c} / \omega_{0}=0.2,2,5$ in Table II. The dependence of the roots on $|m|$ is shown both numerically (from Table III) and analytically (from Eq. (2)). As it is clearly seen from Table II, the roots $r_{0}$ increase as $|m|$ decreases and thus the e-e

TABLE II

The values of the roots of $r_{0}$ calculated for quantum states $|0, m\rangle, m=0,-1,-2, \ldots,-10$, for $\ell=3 a^{*}$ and different values of $\omega_{c} / \omega_{0}$ ratio.

\begin{tabular}{l|c|c|c}
\hline \hline$|0, m\rangle$ & $\omega_{\mathrm{c}} / \omega_{0}=0.2$ & $\omega_{\mathrm{c}} / \omega_{0}=2$ & $\omega_{\mathrm{c}} / \omega_{0}=5$ \\
\hline$|0,0\rangle$ & 4.322 & 3.692 & 2.504 \\
$|0,-1\rangle$ & 5.130 & 4.222 & 2.943 \\
$|0,-2\rangle$ & 5.784 & 4.801 & 3.398 \\
$|0,-3\rangle$ & 6.429 & 5.363 & 3.398 \\
$|0,-4\rangle$ & 7.041 & 5.890 & 4.220 \\
$|0,-5\rangle$ & 7.618 & 6.384 & 4.586 \\
$|0,-6\rangle$ & 8.162 & 6.847 & 4.929 \\
$|0,-7\rangle$ & 8.676 & 7.285 & 5.250 \\
$|0,-8\rangle$ & 9.165 & 7.700 & 5.555 \\
$|0,-9\rangle$ & 9.631 & 8.096 & 5.844 \\
$|0,-10\rangle$ & 10.077 & 8.473 & 6.120
\end{tabular}

TABLE III

Numerical analysis for the electron-electron and magneto-confining energy terms for the first and the second transitions which occur in the ground states of the parabolic quantum dot size $\ell_{0}=3 a^{*}$.

\begin{tabular}{r|c|c|c|c|c|c|c|c}
\hline \hline$m$ & $\omega_{\mathrm{c}} / 2$ & $\omega_{\mathrm{c}} / \omega_{0}$ & $\omega$ & $r_{0}$ & $\sqrt{2} / r_{0}$ & $\frac{1}{4} \omega^{2} r_{0}^{2}$ & $E^{(0)}$ & $E$ \\
\hline 0 & 0.0667 & 0.6 & 0.2320 & 4.1250 & 0.3428 & 0.2289 & 0.5717 & 1.1030 \\
-1 & 0.0667 & 0.6 & 0.2320 & 4.7950 & 0.2949 & 0.3093 & 0.6042 & 1.0201 \\
\hline \hline-1 & 0.2000 & 1.8000 & 0.2989 & 4.3440 & 0.3255 & 0.4214 & 0.7469 & 1.1264 \\
-2 & 0.2000 & 1.8000 & 0.2989 & 4.9340 & 0.2866 & 0.5437 & 0.8303 & 1.1387
\end{tabular}


interaction energy, $V_{\mathrm{e}-\mathrm{e}}\left(r_{0}, m\right)=\sqrt{2} / r_{0}(m)$, decreases showing its dependence on the azimuthal quantum number. On the other hand, the magneto-confining energy term $\frac{1}{4} \omega^{2} r_{0}^{2}$ increases. For the particular relative state $|0, m\rangle$ and fixed $\ell \sim \omega_{0}$, the roots $r_{0}$ decrease as we increase the strength of the magnetic field $\sim \omega_{0}$ and so the electron-electron interaction energy $\sim \frac{1}{r_{0}}$ for this state enhances. As we sweep the magnetic field more, the e-e energy increases and thus the electron jumps to the next state with a higher angular momentum $m$, equivalently, larger root of $r_{0}$ and thus the e-e energy in the new state tends to a decrease.

To understand how the second transition occurs at a higher magnetic field comparable to the first transition, we must look to Table III. This shows quantitatively how the competition between the e-e interaction energy and the confinement energy occurs for $m=0,-1,-2$ states. These are calculated at the transition ratios $\omega_{\mathrm{c}} / \omega_{0}=0.5$ and 1.9. Concerning the first transition: the Coulomb energy decreases by 0.04779 whilst the quantum state changes from $m=0$ to -1 . On
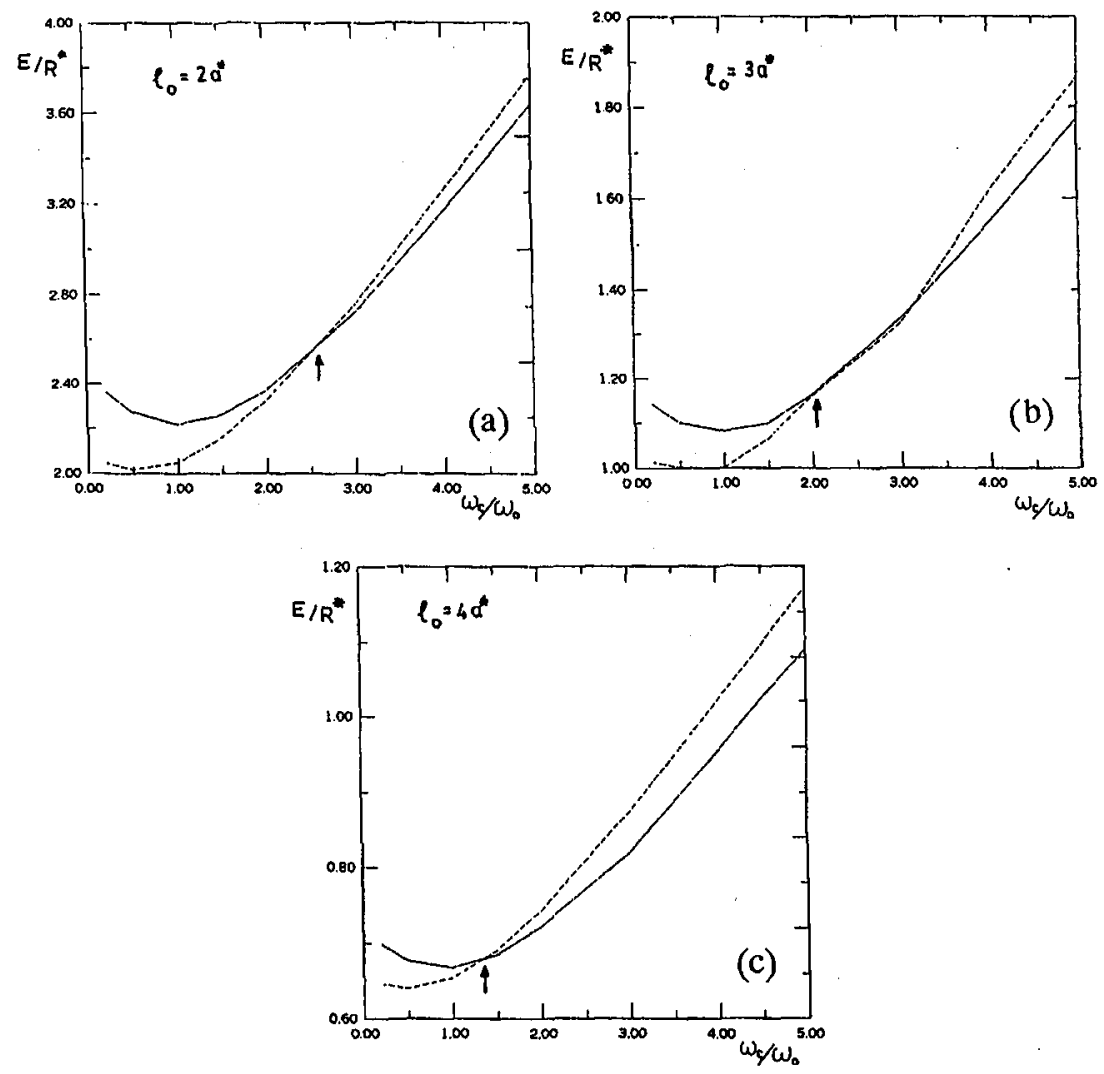

Fig. 2. The total energies of the states $m=-1$ and -2 for two interacting electrons, parabolically confined in quantum dots of sizes: (a) $\ell_{0}=2 a^{*}$, (b) $\ell_{0}=3 a^{*}$, (c) $\ell_{0}=4 a^{*}$, $(---m=-1,-m=-2)$. 
the other hand, the magneto-confining energy increases by 0.0804 . In the second transition: the interaction energy decreases by 0.0389 whilst the magneto-confining energy increases by 0.1223 . The enhancement in the magneto-confining energy corresponds to an increase in the effective frequency $\omega=\left[\left(\omega_{\mathrm{c}} / 2 \omega_{0}\right)^{2}+1\right]^{1 / 2} \omega_{0}$. For the particular quantum dot size $\ell=\left(\hbar / m^{*} \omega_{0}\right)^{1 / 2}$, this means that the applied magnetic field should be high in order the second transition to be observed.

We have plotted, in Fig. 2 and also listed in Table IV the calculated energies of the states $m=-1$ and -2 as a function of the ratio $\omega_{\mathrm{c}} / \omega_{0}$ for quantum dots

\section{TABLE IV}

The energies of the relative states $m=0,-1,-2$ calculated for quantum dot of various sizes: $\ell_{0}=$ $3 a^{*}$ and $4 a^{*}$. The stars show the $\omega_{c} / \omega_{0}$ ratio, where the transition occurs and the arrows indicate the corresponding energies of the quantum dot states: $|0,0 ; 0,-1\rangle$ and $|0,0 ; 0,-2\rangle$.

\begin{tabular}{l|c|c|c|c}
\hline \hline$\omega_{\mathrm{c}} / \omega_{0}$ & \multicolumn{2}{|c|}{$\ell_{0}=2 a^{*}$} & \multicolumn{2}{c}{$\ell_{0}=4 a^{*}$} \\
\cline { 2 - 5 } & $m=-1$ & $m=-2$ & $m=-1$ & $m=-2$ \\
\hline 0.2 & 2.045 & 2.366 & 0.643 & 0.699 \\
0.5 & 2.014 & 2.274 & 0.640 & 0.677 \\
1.0 & 2.042 & 2.218 & 0.654 & 0.669 \\
$1.5^{*}$ & 2.149 & 2.260 & 0.689 & 0.686 \\
2.0 & 2.311 & 2.372 & 0.740 & 0.722 \\
$3.0^{*}$ & 2.731 & 2.721 & 0.828 & 0.820 \\
4 & 3.219 & 3.159 & 1.013 & 0.947 \\
5 & 3.737 & 3.641 & 1.165 & 1.092
\end{tabular}

\section{TABLE V}

The energies of the states $\left|n_{r}, m ; n_{\mathrm{cm}}, m_{\mathrm{cm}}, s\right\rangle$ calculated at $\gamma=\omega_{\mathrm{c}} / 2=1$ by the $1 / D$ expansion method and numerical results Ref. [15].

\begin{tabular}{c|c|c}
\hline \hline$\left|n_{r}, m ; n_{\mathrm{cm}}, m_{\mathrm{cm}},\right\rangle$ & Ref. [15] & $(1 / D$ expansion $)$ \\
\hline$|0,1 ; 0,0,1\rangle$ & 3.8278 & 3.7953 \\
$|0,2 ; 0,0,0\rangle$ & 4.6436 & 4.6432 \\
$|0,3 ; 0,0,1\rangle$ & 5.5174 & 5.5136 \\
$|0,2 ; 0,1,0\rangle$ & 5.6436 & 5.6432 \\
$|0,4 ; 0,0,0\rangle$ & 6.4693 & 6.4782 \\
$|1,2 ; 0,0,0\rangle$ & 6.5956 & 6.5844 \\
$|0,2 ; 1,0,0\rangle$ & 6.6436 & 6.6432
\end{tabular}



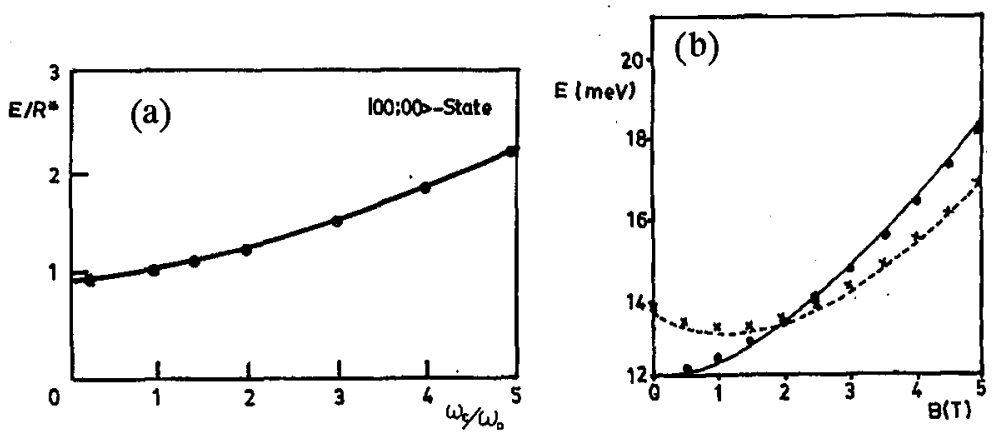

Fig. 3. (a) The total ground-state energy $|0,0 ; 0,0\rangle$ for two interacting electrons in a quantum dot of size $\ell_{0}=3 a^{*}$ against the ratio $\omega_{c} / \omega_{0}$.

Ref. [7]). (b) The eigenenergies of the states $|0,0 ; 0,0\rangle$ (solid curve - exact, black circles - present results) and $|0,0 ; 0,-1\rangle$ (broken curve - exact; crosses - present results) against the magnetic field for $\hbar \omega_{0}=3 \mathrm{meV}$. The exact diagonalizations are taken from Refs. $[8,11]$.

with different sizes $\ell_{0}=2 a^{*}, 3 a^{*}$, and $4 a^{*}$. The figures clearly show the influence of the dot sizes on the transition ratio. We found that the transition ratios occur at 3,1.9, and 1.5. Our results are also in agreement with Ref. [15]. In addition to the qualitative agreement, in Table $\mathrm{V}$ we have compared our computed energy spectra with those of Zhu et al. [15] calculated at $\gamma=\omega_{c} / 2=1$. These tabulated results also show very good quantitative agreement. In addition to this, we have also compared our results with the exact ones given in Refs. $[7,8,11]$. Our results displayed in Fig. 1, for $g^{*}=0$, matches the corresponding one in Fig. 1b of Ref. [7]. In Fig. 3a, b we have shown our results separately, for particular quantum states, produced by the $1 / D$ expansion method against the exact ones $[8,11]$. The figures show that our results agree very well with the numerical data.

\section{Conclusion}

We have used the shifted $1 / D$ expansion method to calculate the spectra of the parabolic quantum dot. The spin singlet-triplet transition, which occurs in the ground state of the quantum dot; is explained. We have also shown the influence of the quantum dot size on the transition ratios. Based on the calculated results, the shifted $1 / D$ expansion method is an effective tool of producing and understanding the spectral properties of the two-electron QD presented in a magnetic field of arbitrary strength.

\section{Appendix}

The parameters $\alpha_{1}$ and $\alpha_{2}$, appeared in Eq. (7), are given as follows:

$$
\begin{aligned}
\alpha_{1}= & {\left[\left(1+2 n_{r}\right) e_{2}+3\left(1+2 n_{r}+2 n_{r}^{2}\right) e_{4}\right] } \\
& -\bar{\omega}^{-1}\left[e_{1}^{2}+6\left(1+2 n_{r}\right) e_{1} e_{3}+\left(11+30 n_{r}+30 n_{r}^{2}\right) e_{3}^{2}\right],
\end{aligned}
$$




$$
\begin{aligned}
\alpha_{2}= & \left(1+2 n_{r}\right) d_{2}+3\left(1+2 n_{r}+2 n_{r}^{2}\right) d_{4}+5\left(3+8 n_{r}+6 n_{r}^{2}+4 n_{r}^{3}\right) d_{6} \\
& -\bar{\omega}^{-1}\left[\left(1+2 n_{r}\right) e_{2}^{2}+12\left(1+2 n_{r}+2 n_{r}^{2}\right) e_{2} e_{4}+2 e_{1} d_{1}\right. \\
& +2\left(21+59 n_{r}+51 n_{r}^{2}+34 n_{r}^{3}\right) e_{4}^{2}+6\left(1+2 n_{r}\right) e_{1} d_{3} \\
& +30\left(1+2 n_{r}+2 n_{r}^{2}\right) e_{1} d_{5}+6\left(1+2 n_{r}\right) e_{3} d_{1} \\
& \left.+2\left(11+30 n_{r}+30 n_{r}^{2}\right) e_{3} d_{3}+10\left(13+40 n_{r}+42 n_{r}^{2}+28 n_{r}^{3}\right) e_{3} d_{5}\right] \\
& +\bar{\omega}^{-2}\left[4 e_{1}^{2} e_{2}+36\left(1+2 n_{r}\right) e_{1} e_{2} e_{3}+8\left(11+30 n_{r}+30 n_{r}^{2}\right) e_{2} e_{3}^{2}\right. \\
& +24\left(1+n_{r}\right) e_{1}^{2} e_{4}+8\left(31+78 n_{r}+78 n_{r}^{2}\right) e_{1} e_{3} e_{4} \\
& \left.+12\left(57+189 n_{r}+225 n_{r}^{2}+150 n_{r}^{3}\right) e_{3}^{2} e_{4}\right] \\
& -\bar{\omega}^{-3}\left[8 e_{1}^{3}+108\left(1+2 n_{r}\right) e_{1}^{2} e_{3}^{2}+48\left(11+30 n_{r}+30 n_{r}^{2}\right) e_{1} e_{3}^{3}\right. \\
& \left.+30\left(31+109 n_{r}+141 n_{r}^{2}+94 n_{r}^{3}\right) e_{3}^{4}\right]
\end{aligned}
$$

with

$$
e_{j}=\varepsilon_{j} / \bar{\omega}^{j / 2} \text { and } d_{i}=\delta_{i} / \bar{\omega}^{i / 2} \text {, }
$$

where $j=1,2,3,4, i=1,2,3,4,5,6$.

The definition of $\varepsilon_{j}$ and $\delta_{i}$ quantities are

$$
\begin{aligned}
& \varepsilon_{1}=(2-a), \quad \varepsilon_{2}=-3(2-a) / 2, \quad \varepsilon_{3}=-1+r_{0}^{5} V^{(3)}\left(r_{0}\right) / 6 Q, \\
& \varepsilon_{4}=\frac{5}{4}+\frac{r_{0}^{6} V^{(4)}\left(r_{0}\right)}{24 Q}, \quad \delta_{1}=-(1-a)(3-a) / 2, \quad \delta_{2}=3(1-a)(3-a) / 4, \\
& \delta_{3}=2(2-a), \quad \delta_{4}=-5(2-a) / 2 .
\end{aligned}
$$

\section{References}

[1] H. Drexler, D. Leonard, W. Hansen, J.P. Kotthaus, P.M. Petroff, Phys. Rev. Lett. 73, 2252 (1994).

[2] C. Sikorski, U. Merkt, Phys. Rev. Lett. 62, 2164 (1989).

[3] T. Demel, D. Heitmann, P. Grambow, K. Ploog, Phys. Rev. Lett. 64, 788 (1990).

[4] A. Lorke, J.P. Kothaus, K. Ploog, Phys. Rev. Lett. 64, 2259 (1990).

[5] R.C. Ashoori, H.L. Stormer, J.S. Weiner, L.N. Pfeiffer, K.W. Baldwin, K.W. West, Phys. Rev. Lett. 71, 613 (1993).

[6] P.A. Maksym, T.T. Chakraborty, Phys. Rev. Lett. 65, 108 (1990).

[7] M. Wagner, U. Merkt, A.V. Chaplik, Phys. Rev. B 45, 1951 (1992).

[8] D. Pfannkuche, R.R. Gerhardts, Physica B 189, 6 (1993).

[9] J.J.S. De Groote, J.E.M. Honos, A.V. Chaplik, Phys. Rev. B 46, 12773 (1992).

[10] U. Merkt, J. Huser, M. Wagner, Phys. Rev. B 43, 7320 (1991).

[11] D. Pfannkuche, R.R. Gerhardts, Phys. Rev. B 44, 13132 (1991).

[12] K.D. Zhu, S.W. Gu, Phys. Lett. A 172, 296 (1993). 
[13] N.F. Jhonson, M.C. Payne, Phys. Rev. Lett. 67, 1157 (1991).

[14] S. Klama, E.G. Mishchenko, J. Phys., Condens. Matter 10, 3411 (1998).

[15] J.L. Zhu, Y. Kawazoe, T. Yao, J. Phys., Condens. Matter 11, 229 (1999).

[16] E. Anisimovas, A. Matulis, J. Phys., Condens. Matter 10, 601 (1998).

[17] A. Matulis, P.M. Peeters, J. Phys., Condens. Matter 6, 7751 (1994).

[18] Y.M. Blanter, N.E. Kaputkina, Y.E. Lozovik, Physica Scr. 54, 539 (1996).

[19] F.M. Peeters, V.A. Schweigert, Phys. Rev. B 53, 1468 (1996).

[20] J.H. Oh, K.J. Chang, G. Ihm, S.J. Lee, Phys. Rev. B 53, 13264 (1996).

[21] T. Imbo, A. Pagnamento, U. Sukhatme, Phys. Rev. D 29, 8763 (1984).

[22] T. Imbo, U. Sukhatme, Phys. Rev. D 28, 418 (1983); 31, 2655 (1985).

[23] R. Dutt, U. Mukherji, Y.P. Varshni, J. Phys. B 19, 3411 (1986).

[24] M. El-Said, Semicond. Sci. Technol. 10, 1310 (1995). 